\title{
A NOTE ON RINGS WHICH ARE MULTIPLICATIVELY GENERATED BY IDEMPOTENTS AND NILPOTENTS
}

\author{
HAZAR ABU-KHUZAM \\ DPp -rtment of Mathematical Sciences \\ University of Petroleum and Minerals \\ Dhahran, Saudi Arabia \\ (Received June 2, 1986 and in revised form September 16, 1986)
}

ABSTRACT. We give the structure of certain rings which are multiplicatively generated by nilpotents or multiplicatively generated by idempotents and nilpotents.

KEY WORDS AND PHRASES. Boolean ring, Nil ring, polynomial identity. 1980 AMS SUBJECT CLASSIFICATION CODE. 16 A70.

1. INTRODUCTION.

In a Boolean ring, every element is trivially a product of idempotents. On the other hand, in a nil ring, every element is trivially a product of nilpotents. This motivates the study of the structure of a ring, which as a semi-group, is generated by its idempotents, or is generated by its nilpotents, or more generally, is generated by its idempotents and nilpotents. Indeed, we prove that a ring which is multiplicatively generated by its nilpotents is nil if it is Artinian or if it satisfies the polynomial identity $x^{m}=x^{m+1} f(x) \quad(f(x)$ is a polynomial with integer coefficients). We also prove that if $R$ is a ring which is multiplicatively generated by its idempotents and nilpotents such that the set $\mathrm{N}$ of nilpotent elements is commutative, then $N$ forms an ideal of $R$ and $R / N$ is Boolean. We also give examples to show that our conditions are essential for the validity of our theorems.

We start with the following definitions, the first of which was introduced in [1]. DEFINITIONS. A ring $R$ is called an I-ring if as a semigroup $R$ is generated by its idempotents. A ring $R$ is called an $\mathrm{N}$-ring if as a semi-group $R$ is generated by its nilpotents. $R$ is said to be an NI-ring if as a semigroup $R$ is generated by its idempotents and nilpotents.

The following two theorems were proved in [1].

THEOREM A. Let $R$ be an I-ring with identity. Then $R$ is Boolean.

THEOREM B. Let $R$ be a finite I-ring. Then $R$ is Boolean.

REMARKS.

1. A homomorphic image of an I-ring, N-ring, or an NI-ring is an I-ring, N-ring, or an NI-ring.

2. If $R$ is an $N$-ring with identity, then $R=\{0\}$.

3. Trivially, every I-ring and every N-ring is an NI-ring. 
4. An I-ring need not be Boolean as shown in [1]. An N-ring need not be nil (see Example 1 below). An NI-ring need not be neither Boolean nor nil (see Example 2 below).

2. MAIN RESULTS.

In preparation for the proofs of our theorems, we start with the following lemmas. Lemma 1 is known but we give its proof for completeness.

LEMMA 1. Let $R$ be a ring such that for some positive integer $\mathrm{m}$, and some polynomial $f(x)$ with integer coefficients, $x^{m}=x^{m+1} f(x)$ for all $x$ in $R$. Then $=x^{m}(f(x))^{m}$ is an idempotent of $R$ for all $x$ in $R$. PROOF. $\left.x^{m}=x^{m+1} f(x)=x^{m} x f(x)=x^{m+2} f(x)\right)$. Continuing we get $x^{m}=x^{2 m}$ $(f(x))^{m}$ which implies that $e=x^{m}(f(x))^{m}$ is an idempotent.

LEMMA 2. If a ring $R$ satisifes the polynomial identity $x^{m}=x^{m+1} f(x)$, then the Jacobson radical $\mathrm{J}$ of $\mathrm{R}$ is nil.

PROOF. Let $x \in J$. By Lemma $1, x^{m}(f(x))^{m}$ is an idempotent element in $J$. So $x^{m}(f(x))^{m}=0$ and since $x^{m}=x^{2 m}(f(x))^{m}$ (Lemma 1), we obtain $x^{m}=0$ for every $x$ in $J$. So $H$ is nil.

In $\{1\rfloor$, it is proved that a finite I-ring is Boolean. In the following two theorems we study the analogous case for N-rings. Indeed, we prove that an $N-r i n g ~ R$ is nil of $R$ is Artinian or if $R$ satisfies the polynomial identity $x^{m}=x^{m+1} f(x)$.

THEOREM 1. Let $R$ be an Artinian N-ring. Then $R$ is nilpotent.

PROOF. Let $J$ be the Jacobson radical of $R$. Suppose $J \neq R$, then $R / J$ (being semisimple Artinian) has an identity. So $\mathrm{R} / \mathrm{J}$ is an $\mathrm{N}-$ ring with identity (Remark 1 ). Thus $R / J=\{0\}$, by Remark 2. This contradicts our assumption that $J \neq R$. So $\mathrm{R}=\mathrm{J}$, and hence $\mathrm{R}$ is nilpotent, since $\mathrm{J}$ is nilpotent in an Artinian ring.

THEOREM 2. Let $R$ be an $N$-ring satisfying the polynomial identity $x^{m}=x^{m+1} f(x)$ ( $\mathrm{m}$ is a positive and $\mathrm{f}(\mathrm{x})$ is a polynomial with integer coefficients). Then $R \underline{\text { is }}$ ni1.

PROOF. By Lemma 2, the Jacobson radical $J$ of $R$ is nil. $R / J$ being semisimple is semiprime, and hence $R / J$ is a subdirect product of prime rings $R_{\alpha}$. Each nonzero prime ring $R_{\alpha}$ satisfies the identity $x^{m}=x^{m+1} f(x)$, and hence by Theorem 1.4.2 of [2], $R_{\alpha}$ has a nontrivial center. Let $c_{\alpha} \neq 0$ be a central element of $R_{\alpha}$. By Lemma $1, e_{\alpha}=c_{\alpha}^{m}\left(f\left(c_{\alpha}\right)\right)^{m}$ is an idempotent of $R_{\alpha}$, and hence $e_{\alpha}$ is a central idempotent of $R_{\alpha} \cdot e_{\alpha} \neq 0$, otherwise $c_{\alpha}^{m}=c_{\alpha}^{2 m}\left(f\left(c_{\alpha}\right)\right)^{m}=0$ which contradicts the fact that $c_{\alpha}$ is a nonzero central element of a prime ring and cannot be a zero divisor by Lemma 2.1 .3 of [3]. But $e_{\alpha} R_{\alpha}\left(e_{\alpha} x_{\alpha}-x_{\alpha}\right)=0$ for all $x_{\alpha} \epsilon R_{\alpha}$. So $e_{\alpha} x_{\alpha}-x_{\alpha}=0$ for all $x_{\alpha}$ in $R_{\alpha}$, and hence $R_{\alpha}$ has an identity element. So $R_{\alpha}$ is an N-ring (Remark 1) with identity. So $R_{\alpha}=0$ (Remark 2). This implies that $\mathrm{R} / \mathrm{J}=\{0\}$, and $\mathrm{R}=\mathrm{J}$ is nil.

We now give an example to show that Theorem 1 need not be true if $R$ is not Artinian and Theorem 2 need not be true if $R$ does not satisfy the identity $x^{m}=x^{m+1} f(x)$. The ring used in the following example was used in [1] to show that an I-ring need not be Boolean.

EXAMPLE 1. Let $D$ be any ring with identity, and let $R$ be the ring of all $\infty \times \infty$ matrices over $\mathrm{D}$ in which at most a finite number of entries are nonzero. Let $x$ be any element of $R$. Then, for some positive integer $n$ and some $n \times n$ matrix 
$A$ over $D$ we have

$$
\mathrm{X}=\left[\begin{array}{ll}
\mathrm{A} & 0 \\
0 & 0
\end{array}\right] ; \mathrm{A} \text { is } \mathrm{n} \times \mathrm{n}, \quad 0^{\prime} \mathrm{s} \text { are zero matrices. }
$$

Let $S=\left[\begin{array}{lll}{ }^{0} & I_{n} & 0 \\ 0 & 0 & 0\end{array}\right] ; \quad T=\left[\begin{array}{ll}O_{n} & 0 \\ A & 0 \\ 0 & 0\end{array}\right] ; \quad 0$ 's are zero matrices.

It is easy to verify that $S$ and $T$ are nilpotent elements, and $X=S T$. Thus $R$ is an N-ring which is not nil since $\left[\begin{array}{ll}1 & 0 \\ 0 & 0\end{array}\right]$ is not nilpotent. This example shows that we cannot drop the hypothesis that $R$ is Artinian in Theorem 1 or the hypothesis that $R$ satisfies the identity $x^{m}-x^{m+1} f(x)$ in Theorem 2 .

Next we study the structure of certain NI-rings. The following example shows that an NI-ring need not be neither Boolean nor nil.

$$
\text { Example 2. Let } R=\left[\begin{array}{ll}
0 & 0 \\
0 & 0
\end{array}\right],\left[\begin{array}{ll}
1 & 0 \\
1 & 0
\end{array}\right],\left[\begin{array}{ll}
0 & 1 \\
0 & 1
\end{array}\right],\left[\begin{array}{ll}
1 & 1 \\
1 & 1
\end{array}\right]
$$

over GF(2). Trivially, $\mathrm{R}$ is a finite NI-ring which is neither Boolean nor nil.

In example 2 above, the NI-ring $R$ has the property that the set $N$ of nilpotent elements forms an ideal of $R$ and $R / N$ is Boolean. This motivates the study in the next theorem. Indeed, we prove that an NI-ring will have this property if the nilpotent elements of $R$ commute.

THEOREM 3. Let $R$ be an NI-ring such that the set $N$ of nilpotent elements of $R$ is commutative. Then $N$ is an ideal of $R$ and $R / N$ is Boolean.

PROOF. If $R$ has no nonzero idempotents, then $R$ is multiplicatively generated by nilpotents only. So $\mathrm{R}=\mathrm{N}$ is nil since $\mathrm{N}$ is commutative, and the theorem follows. So we may assume that $R$ has nonzero idempotents. Let $e$ be any nonzero idempotent of $R$ and let $x$ be any element of $R$. Clearly, (ex - exe) $\epsilon$ and (xe - exe) $\epsilon \mathrm{N}$. Now, since $\mathrm{N}$ is commutative

$$
e(e x-e x e)(x e-e x e)=e(x e-e x e)(e x-e x e)=0
$$

This implies that $\mathrm{ex}^{2}-$ exexe $=0$, and hence

(1) $(\text { exe })^{2}=e x^{2} e$.

Using induction, (1) implies that

(2) $(e x e)^{2^{n}}=e^{2^{n}}$ e for all positive integers $n$.

Let a $\in \mathrm{N}$. Then using (2) we obtain

(3) eae $\epsilon \mathrm{N}$ for every a $\epsilon \mathrm{N}$.

Since $N$ is commutative, $N$ is a subring of $R$. So using (3) and the fact that ea - eae $\epsilon \mathrm{N}$ and ae - eae $\epsilon \mathrm{N}$ we get 
(4) ea $\epsilon N$, ae $\epsilon N$ for every a $\epsilon N$ and every idempotent e.

Now since $\mathrm{R}$ is multiplicatively generated by idempotents and nilpotnets and since $\mathrm{N}$ is commutative, (4) implies that

(5) $\mathrm{N}$ is an ideal of $\mathrm{R}$.

Let $\bar{x}=x+N$ be any nonzero element of $R / N$. Since $R$ is an NI-ring, (5) implies that either $x \in N$ or $x=e_{1} e_{2} \ldots e_{n}$ for some idempotent elements

$e_{1}, e_{2}, \ldots, e_{n}$. So

$$
\bar{x}=e_{1} e_{2} \ldots e_{n}+N=\left(e_{1}+N\right)\left(e_{2}+N\right) \ldots\left(e_{n}+N\right)
$$

and hence

(6) $\mathrm{R} / \mathrm{N}$ is an I-ring.

If $\bar{e}$ is any idempotent element of $R / N$, then $(\bar{e} \bar{x}-\bar{e} \bar{e})$ and $(\bar{x} \bar{e}-\bar{e} \bar{x})$ are nilpotent elements of $\mathrm{R} / \mathrm{N}$. But $\mathrm{R} / \mathrm{N}$ has no nonzero nilpotent elements. Thus $\overline{\mathrm{e}} \overline{\mathrm{x}}=\overline{\mathrm{e}} \overline{\mathrm{x}} \overline{\mathrm{e}}=\overline{\mathrm{x}} \overline{\mathrm{e}}$ for $\mathrm{all} \mathrm{x}$ in $\mathrm{R} / \mathrm{N}$ and hence

(7) The idempotents of $\mathrm{R} / \mathrm{N}$ are central.

Now, by (6) and (7), $\mathrm{R} / \mathrm{N}$ is I-ring with central idempotent elements, and hence $\mathrm{R} / \mathrm{N}$ is Boolean. This completes the proof of Theorem 3 .

We now give an example to show that Theorem 3 need not be true if the nilpotents of $R$ do not commute.

EXAMPLE 3. Let $R$ be the ring of Example 1. Then $R$, being an $N-r i n g$, is an NI-ring. Clearly, the set $N$ of nilpotent elements of $R$ is not an ideal of $R$. This example shows that we cannot drop the hypothesis that the nilpotent commute in Theorem 3.

\section{REFERENCES}

1. PUTCHA, M.S. and YAQUB, A. "Rings which are Multiplicatively Generated by Idempotents," Communications in Algebra 8(1980), 153-159.

2. HERSTEIN, I.N. Rings With Involution, University of Chicago Press, Chicago, 1976.

3. HERSTEIN, I.N. Noncommutative Rings, The Carus Math. Monographs, John Wiley and Sons, Inc., 1968. 


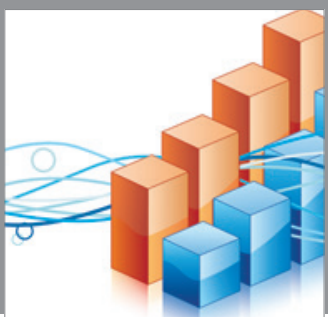

Advances in

Operations Research

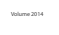

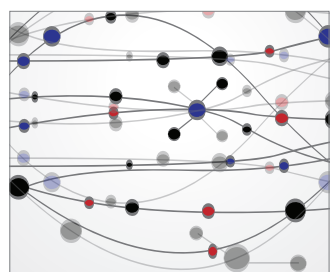

\section{The Scientific} World Journal
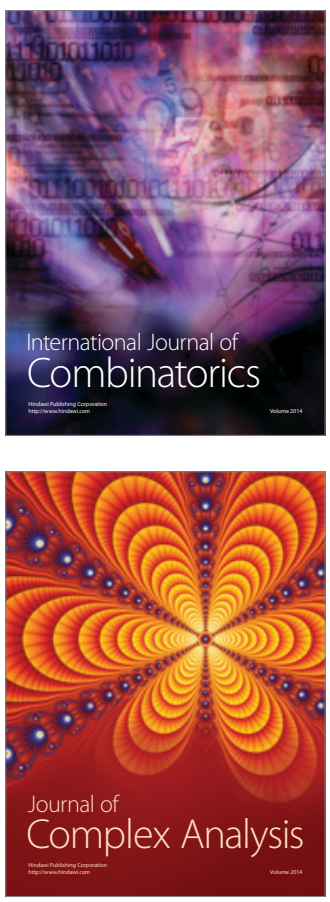

International Journal of

Mathematics and

Mathematical

Sciences
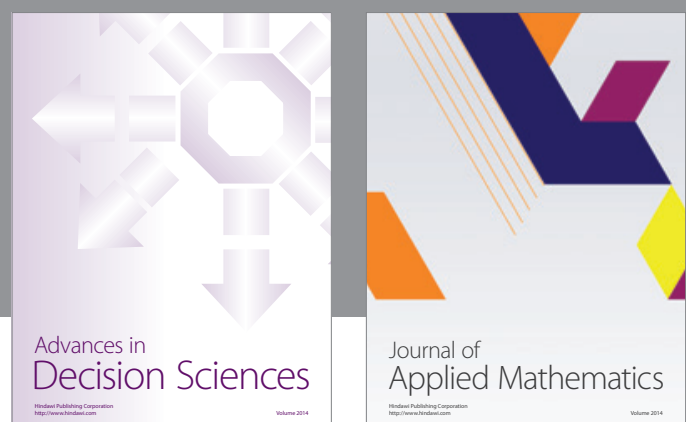

Journal of

Applied Mathematics
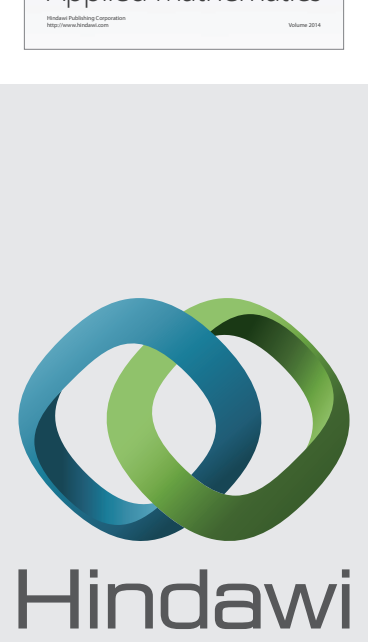

Submit your manuscripts at http://www.hindawi.com
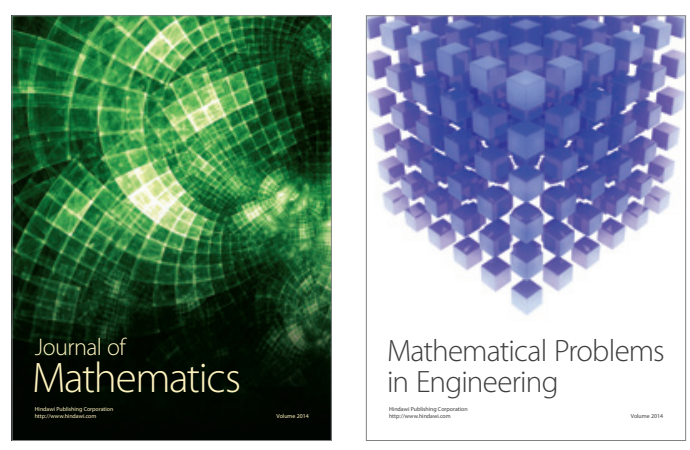

Mathematical Problems in Engineering
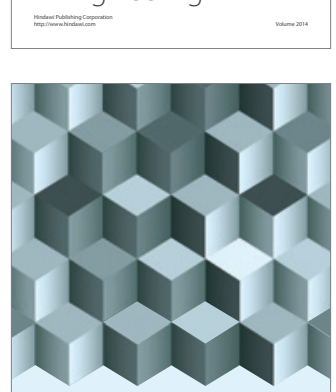

Journal of

Function Spaces
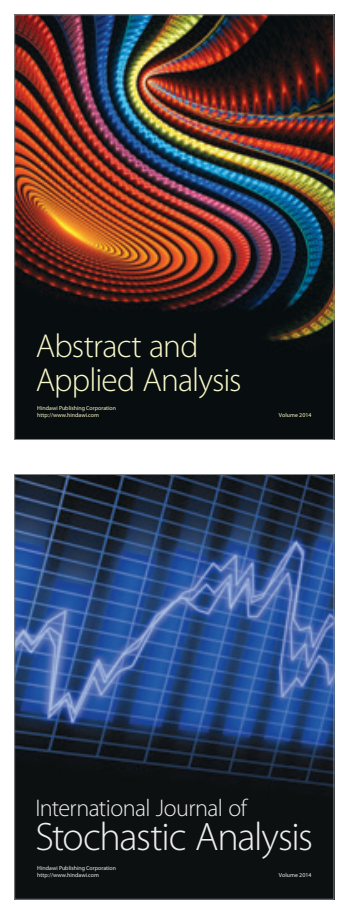

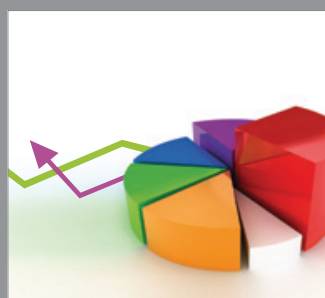

ournal of

Probability and Statistics

Promensencen
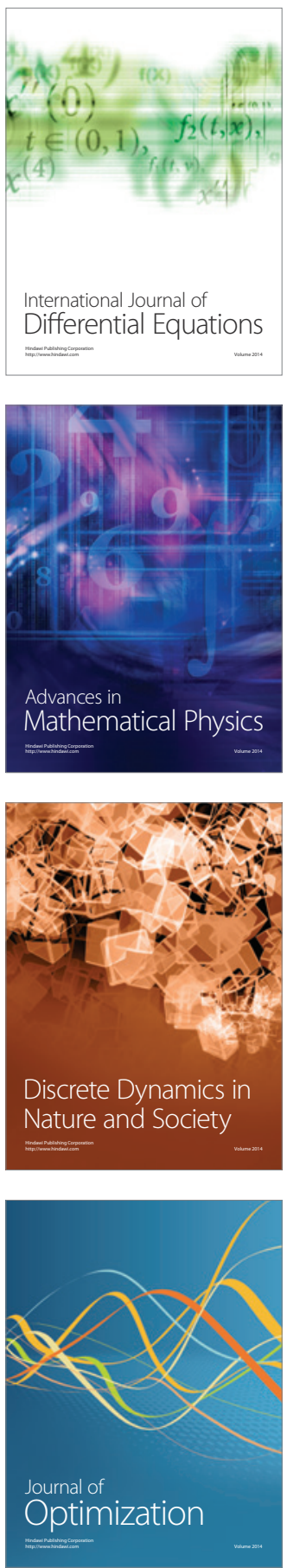